\title{
Early pathological gambling in co-occurrence with semantic variant primary progressive aphasia: a case report
}

This article was published in the following Dove Press journal: Clinical Interventions in Aging

\author{
Petronilla Battista ${ }^{1,2}$ \\ Rosa Capozzo ${ }^{2}$ \\ Giovanni Rizzo 3,4 \\ Chiara Zecca ${ }^{2}$ \\ Antonio Anastasia ${ }^{5}$ \\ Roberto De Blasi ${ }^{6}$ \\ Giancarlo Logroscino ${ }^{2,7}$ \\ 'Istituti Clinici Scientifici Maugeri-SPA SB. \\ I.R.C.C.S. Institute of Cassano Murge, \\ Bari, Italy; ${ }^{2}$ Neurodegenerative Disease \\ Unit, Department of Clinical Research in \\ Neurology, University of Bari Aldo Moro, \\ "Pia Fondazione Cardinale G. Panico", \\ Tricase, Italy; ${ }^{3} \mathrm{UOC}$ Clinica Neurologica, \\ IRCCS Istituto Delle Scienze \\ Neurologiche di Bologna, Bologna, Italy; \\ ${ }^{4}$ Department of Biomedical and \\ Neuromotor Sciences, University of \\ Bologna, Bologna, Italy; ${ }^{5}$ Department of \\ Nuclear Medicine, Pia Fondazione di \\ Culto e Religione "Card.G.Panico", \\ Tricase, Italy; ${ }^{6}$ Department of Diagnostic \\ Imaging, Pia Fondazione di Culto e \\ Religione "Card.G.Panico", Tricase, Italy; \\ ${ }^{7}$ Department of Basic Medical Sciences, \\ Neuroscience and Sense Organs, \\ University of Bari Aldo Moro, Bari, Italy
}

Correspondence: Giancarlo Logroscino Department of Clinical Research in Neurology, University of Bari Aldo Moro,

"Pia Fondazione Cardinale G. Panico", Via

S. Pio X, n. 4., Tricase, Lecce 73039, Italy

Tel +39083 3773904

Fax $+390833 \quad 1830670$

Email giancarlo.logroscino@uniba.it

\begin{abstract}
We have comprehensively documented a case of semantic variant of primary progressive aphasia (sv-PPA) presenting with early-onset pathological gambling (PG). While a growing number of studies have shown the presence of behavioral alterations in patients with sv-PPA, PG has been observed only in the behavioral variant of frontotemporal dementia (bv-FTD). To date, no case of PG with the co-occurrence of prominent semantic deficits at the onset of the disease has been reported in the literature. Impulse disorders at onset may wrongly lead to a misdiagnosis (ie, psychiatric disorders). Therefore, a wider characterization of cognitive/aphasia symptoms in patients presenting impulse disorders and predominant language dysfunctions is recommended.
\end{abstract}

Keywords: impulse disorders, language disorders, neurodegenerative diseases, semantic dementia

\section{Introduction}

Semantic variant of primary progressive aphasia (sv-PPA), also known as "semantic dementia", is one of the main clinical variants of frontotemporal dementia (FTD). From a clinical-pathological point of view, FTD degeneration is usually caused by TAR-DNA-binding protein (TDP-43), a 43 kilodaltons that has been reported as the underlying cause of the disease in approximately $68 \%-80 \%$ of subjects with sv-PPA. According to the current diagnostic criteria, ${ }^{2}$ in order to identify patients with sv-PPA, two core features are necessary: the impaired naming of objects more than actions and impaired single-word comprehension. Moreover, several studies have demonstrated that the clinical features of sv-PPA may depend on the atrophy prevalently involved in the left hemisphere rather than in the right one. ${ }^{3}$ For example, severe anomia and impaired single-word difficulties are more likely to be present in patients with a prevalently left hemisphere involvement, ${ }^{4}$ while impaired recognition of familiar and famous faces is a typical consequence of predominantly right hemisphere atrophy. ${ }^{5}$ There is growing awareness of the presence of behavioral alterations in patients with sv-PPA. The most frequent clinical features are disinhibition, impulsivity, bizarre food choices/hyperphagia and repetitive/compulsive behavior that belong to the sphere of positive symptoms. ${ }^{6,7}$ To date, impulse control disorder or, more specifically, pathological gambling (PG) has been widely observed in the behavioral variant of FTD (bvFTD), ${ }^{8,9}$ but no cases of PG with the co-occurrence of semantic deficits at the onset of the disease have been reported in the literature. In this report, we describe the 
case of a 64-year-old male with symptoms of PG and the onset of co-occurrence of semantic impairment typical of sv-PPA.

\section{Case report}

In October 2016, an Italian-speaking, right-handed 64-yearold male, with only eight years of formal education, was referred to our Neurodegenerative Diseases Centre by his family. He presented with a 2-year history of progressive language deficits and impulse control disorders with PG evident from the onset. Before developing PG, the patient was described by his family as being parsimonious and careful with money. Over the two years, he had started to spend his money on gaming machines and scratch cards, squandering his salary and his savings and borrowing money from friends and relatives to continue gambling. From the onset of the disease, the patient also presented semantic deficits and started to use objects inappropriately because he seemed not to recognize them. At initial neurological examination, the patient presented with mild signs of Parkinson's (ie, bradykinesia, hypomimia) and there was a family history of dementia (his father had Alzheimer's disease). In the following month, the patient underwent a neuropsychological assessment that showed an entirely normal cognitive performance, even for executive tasks, with the exception of a slight but not significant impairment in verbal episodic memory that could be related to his lexical-semantic deficits. Activities of daily living were preserved. At the speech-language examination, he showed isolated semantic deficits, ie, impairment in single-word comprehension, nonverbal semantic association, surface dyslexia and word-finding difficulties as assessed by the Screening for Aphasia in Neurodegeneration battery (SAND). ${ }^{10,11}$ At the spontaneous speech task, he showed a low speech rate associated with several false starts and a lot of lexical-semantic errors, ie, word-finding pauses with a tendency to produce high-frequency nouns and poor content resulting in a reduction of information units. The patient's linguistic profile did not report articulatory, phonetic and phonemic errors. PG was confirmed from the Barratt Impulsiveness Scale ${ }^{12}$ and South Oaks Gambling Screen ${ }^{13}$ (Tables 1 and 2).

MRI brain scans ( $3 \mathrm{~T}$ ) showed bilateral (right $>$ left) fronto-temporo-insular and cingulate atrophy (Figure 1, Panels A-B-C); SPECT brain scan showed no right temporal lobe perfusion and hypoperfusion of the bilateral frontal lobes and left cingulate (Figure 2) and DAT-SCAN showed damage to the nigrostriatal dopamine system, ie right putamen, caudate nucleus (right $>$ left) (Figure 3).
CSF biomarkers were within the normal range for age (Aß1-42771 pg/mL; tTau $276 \mathrm{pg} / \mathrm{mL}$; nd $42.79 \mathrm{pg} / \mathrm{mL}$; pTau181: f). Imaging and CSF biomarkers indicated a neurodegenerative process that was not due to amyloidosis (Figure 4).

The patient was diagnosed as having sv-PPA according to the classification of Gorno-Tempini et al. ${ }^{2}$

In November 2017, the language deficit worsened to paragrammatic speech, a low speech rate and reduction of information content. Also, his behavioral changes increasingly worsened with apathy, disinhibition, compulsive/ stereotyped behaviors and hyperphagia, as did performance in executive/social cognition abilities, compatible with a frontal lobe dysfunction (Tables 1 and 2).

This clinical picture indicated the presence of a second phenotype, behavioral-frontotemporal dementia (bv-FTD), according to the criteria of Rascovsky et $\mathrm{al}^{14}$ with associated mild parkinsonism. A diagnosis of fronto-temporal lobar degeneration (FTLD) was established according to the Lund-Manchester criteria. ${ }^{15}$

PG was nonresponsive to pharmacological therapy (eg, levodopa) during the course of the disease and was considered a significant clinical finding within the wider spectrum of behavioral symptoms.

\section{Discussion}

From a clinical point of view, our patient's behavioral and cognitive disorders showed similar trajectories. He had bilateral cerebral atrophy that resulted in the overlapping of behavioral and language symptoms. Behavioral symptoms are usually related to right hemispheric atrophy; therefore, as this was prevalent, we should have expected more evident behavioral symptoms compared to semantic deficits. However, behavioral symptoms became more pronounced during the course of the disease.

Other causes of neurodegenerative dementia were excluded, for example, an atypical frontal form of Alzheimer's disease, ${ }^{16}$ as the patient did not show low levels of beta-amyloid at CSF examination. At the second evaluation, the patient presented with dementia, spontaneous parkinsonism and an altered DAT SCAN, but a diagnosis of Lewy body dementia ${ }^{17}$ was excluded because the clinical picture was unremarkable for two core features (hallucinations and fluctuating cognition) and cognitive functions were reasonably preserved compared to behavioral changes. Treatment with levodopa was started only several months after onset and therefore was not responsible for the PG. 
Table I Patient's neuropsychological score and cutoff score

\begin{tabular}{|c|c|c|c|}
\hline Test & First assessment & Follow up (I year) & Cutoff score \\
\hline $\begin{array}{l}\text { Global cognitive functioning } \\
\text { Mini-mental state examination }\end{array}$ & 28 & 27 & $<23.8$ \\
\hline $\begin{array}{l}\text { Memory } \\
\text { Rey auditory verbal learning test } \\
\text {-Immediate recall } \\
\text {-Delayed recall } \\
\text {-Recognition } \\
\text { Rey recall of copy of the Rey-Osterrieth figure } \\
\text { Digit span forward }\end{array}$ & $\begin{array}{l}25 \\
6 \\
15 \\
21,5 \\
6\end{array}$ & $\begin{array}{l}20 \\
5 \\
10 \\
16,5 \\
5\end{array}$ & $\begin{array}{l}<28.53 \\
<4.69 \\
- \\
<9.47 \\
<4.26\end{array}$ \\
\hline $\begin{array}{l}\text { Attention \& executive functions } \\
\text { Frontal assessment battery } \\
\text { - Trial making test A } \\
\text { - Trial making test B } \\
\text { - Trial making test BA } \\
\text { Digit span backward } \\
\text { Stroop color and word test -time } \\
\text { Stroop color and word test -errors } \\
\text { Phonemic (F-A-S) } \\
\text { Semantic verbal fluency }\end{array}$ & $\begin{array}{l}14 \\
53 \\
158 \\
105 \\
4 \\
28 \\
0 \\
25 \\
23\end{array}$ & $\begin{array}{l}12 \\
67 \\
175 \\
108 \\
4 \\
24 \\
0 \\
8 \\
4\end{array}$ & $\begin{array}{l}<12.03 \\
>94 \\
>283 \\
>187 \\
<2.65 \\
>36.92 \\
>4.24 \\
<17.35 \\
<25\end{array}$ \\
\hline $\begin{array}{l}\text { Social cognition } \\
\text { Story-based empathy task }\end{array}$ & 12 & 3 & $<8.29$ \\
\hline $\begin{array}{l}\text { Visuo-spatial abilities } \\
\text { Copy of the Rey-Osterrieth complex figure } \\
\text { Incomplete letters-VOSP battery }\end{array}$ & $\begin{array}{l}30 \\
17\end{array}$ & $\begin{array}{l}32 \\
16\end{array}$ & $\begin{array}{l}<28.88 \\
<16\end{array}$ \\
\hline $\begin{array}{l}\text { Impulse dyscontrol } \\
\text { Barratt impulsiveness scale } \\
\text { South Oaks gambling screen }\end{array}$ & $\begin{array}{l}65 \\
\text { Positive }\end{array}$ & $\begin{array}{l}68 \\
\text { Positive }\end{array}$ & $\begin{array}{l}<69,3 \\
-\end{array}$ \\
\hline $\begin{array}{l}\text { Behavioral scales } \\
\text { Apathy scale } \\
\text { Geriatric depression scale } \\
\text { Neuropsychiatric inventory } \\
\text { Frontal behavioral inventory }\end{array}$ & $\begin{array}{l}2 \\
6 \\
12 \\
10\end{array}$ & $\begin{array}{l}12 \\
11 \\
34 \\
37\end{array}$ & $\begin{array}{l}>11 \\
>10 \\
- \\
-\end{array}$ \\
\hline $\begin{array}{l}\text { Functional autonomy } \\
A D L \\
\text { IADL }\end{array}$ & $\begin{array}{l}6 / 6 \\
7 / 8\end{array}$ & $\begin{array}{l}4 / 6 \\
3 / 8\end{array}$ & - \\
\hline
\end{tabular}

Note: Bold scores are pathological.

Abbreviations: VOSP, Visual Object and Space Perception Battery; ADL, Activities in Daily Living; IADL, Instrumental Activities in Daily Living.

The clinical information collected may also lead to an alternative, possibly even complementary, hypothesis related to the evolution of the patient's symptoms.

As the disease progresses, the symptoms of the different phenotypes of FTLD can converge as an initially focal atrophy becomes more prominent and spreads to affect large areas in the frontal and temporal lobes. Over time, patients manifest global cognitive impairment and motor deficits, including parkinsonism. ${ }^{18}$ In our patient, parkinsonian signs were very slight at the first clinical examination (without DAT-scan evidence), but had worsened by the second examination (with imaging evidence). This hypothesis sits well with previous studies in which early parkinsonism is present in $18 \%-20 \%$ of patients with FTLD and is most often seen in patients with bv-FTD, while it is rarely seen in sv-PPA, only in about $3 \%$ (for a review $\operatorname{see}^{19}$ ). 
Table 2 Patient's speech and language score and cutoff score. Bold scores are pathological

\begin{tabular}{|l|l|l|l|}
\hline Test & First assessment & Follow-up (I year) & Cutoff score \\
\hline Speech-Language & & & \\
SAND & & & \\
$-\quad$ Naming & $\mathbf{7 / 1 4}$ & $\mathbf{3} / \mathbf{4}$ & $\leq 9.969$ \\
$-\quad$ Sentence comprehension & $8 / 8$ & $6 / 8$ & $\leq 6.157$ \\
$-\quad$ Single-word comprehension & $\mathbf{8 / 1 2}$ & $\mathbf{4 / 1 2}$ & $\leq 10.258$ \\
$-\quad$ Repetition of word and nonwords & $10 / 10$ & $10 / 10$ & $\leq 6.349$ \\
$-\quad$ Repetition of sentences & $4 / 6$ & $2 / 6$ & $\leq 2.455$ \\
$-\quad$ Reading & $12 / 16$ & $12 / 16$ & $\leq 10.106$ \\
$-\quad$ Writing & $4 / 6$ & $2 / 6$ & $\leq 2.132$ \\
$-\quad$ Semantic association & $1 / 4$ & $\mathbf{0 / 4}$ & $\leq 1.166$ \\
$-\quad$ Connected speech & $\mathbf{2 / 6}$ & $\mathbf{1 / 6}$ & $\leq 3$ \\
Token test & $34 / 36$ & $30 / 36$ & $<26.25$ \\
CAGI naming & $\mathbf{2 8 / 4 8}$ & $\mathbf{1 8 / 4 8}$ & $<41.48$ \\
CAGl single-word comprehension & $\mathbf{4 2 / 4 8}$ & $\mathbf{3 2 / 4 8}$ & $<47.08$ \\
Pyramid and Palm Tree Test & $\mathbf{3 7 / 5 2}$ & $\mathbf{2 6 / 5 2}$ & $<40.15$ \\
AAT repetition subtest & $142 / 150$ & $135 / 150$ & - \\
ENPA verbal sentence comprehension subtest & $14 / 14$ & $12 / 14$ & $<11.6$ \\
\hline
\end{tabular}

Note: Bold scores are pathological.

Abbreviations: SAND, Screening for Aphasia in Neurodegeneration; AAT, Aachener Aphasie Test; ENPA, Esame Neuropsicologico per l'Afasia.

A

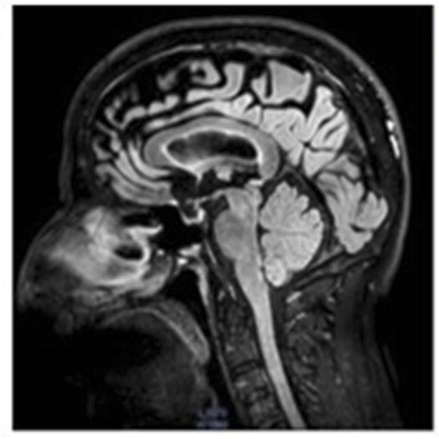

B

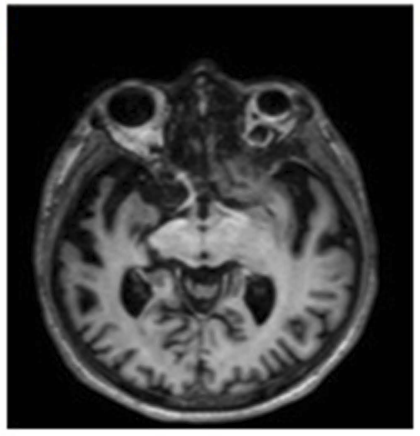

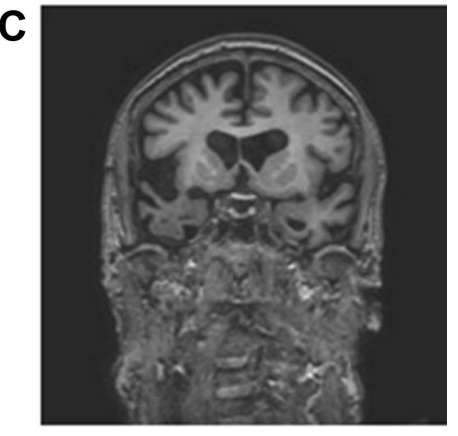

Figure I Neuroimaging examinations. The patient's first MRI brain scan ( 3 tesla), performed about 2 years into his illness. Panel A: Sagittal FLAIR T2 image highlights the marked frontal and cingulate atrophy. Panel B: The axial 3D FFE TI-w image shows bilateral anterior temporal lobe atrophy (right > left). Panel C: Coronal FFE 3D TI-w image shows fronto-insular and anterior temporal cortical atrophy, mainly on the right side.

Abbreviation: MRI, magnetic resonance imaging.

sv-PPA with parkinsonism is not yet fully understood. One study reported that six sv-PPA patients showed parkinsonism, but their clinical features were not defined in detail. ${ }^{20}$ Therefore, the case could be made for a patient with bvFTD and prominent semantic involvement. The parkinsonism seen in bv-FTD patients is usually characterized by akinetic rigid syndrome, ${ }^{21}$ the same kind that we found in our patient. Moreover, specific patterns of cortical and subcortical atrophy in the frontal lobe, insula and anterior cingulate cortex characterize bv-FTD. ${ }^{22,23}$ Our findings in this patient with parkinsonism show a neurodegenerative process involving not only anterior frontal areas, but also posterior frontal regions, including M1 and the corticobasal, ganglia-caudate, nucleus-thalamo-cortical motor loops that are also implied in PG behavior. ${ }^{24}$

At the onset, PG may wrongly lead to a misdiagnosis. It is extremely important to consider several differential diagnoses as these symptoms may overlap with other conditions, ie, late-onset psychiatric disorders. Moreover, prominent PG can lead to an underestimation of cognitive/ language deficits in the early phase of the disease. A wider characterization of impulse disorders in FTLD with predominant language dysfunctions would be crucial for an early diagnosis. 


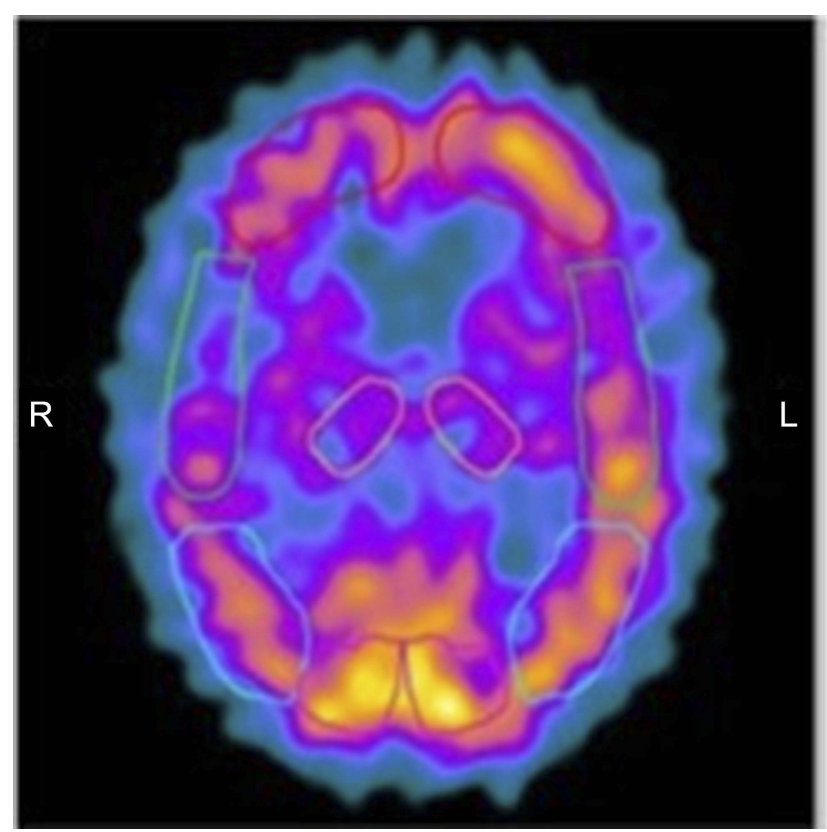

Figure 2 SPECT brain scan shows hypoperfusion of the right temporal lobe and hypoperfusion of the bilateral frontal lobes and left cingulate. Abbreviation: SPECT, single-photon emission computed tomography.

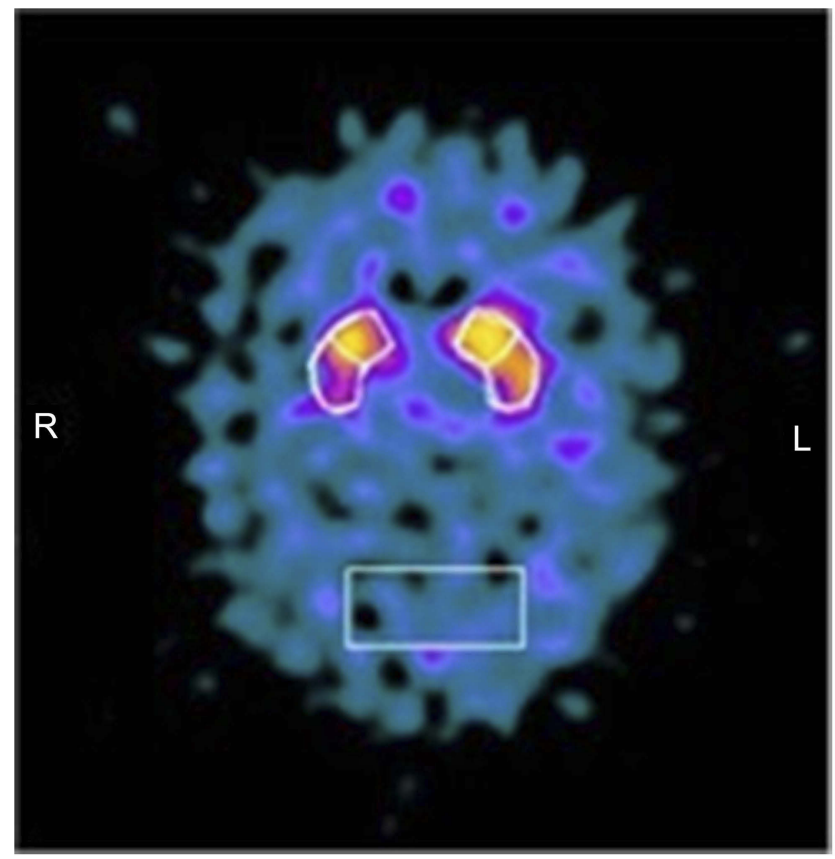

Figure 3 Brain Dat-scan shows damage to the nigrostriatal dopamine system, in particular a loss of dopamine neurons in the right putamen and caudate nucleus (right $>$ left).

However, we are aware that our report presents some limitations. In particular, the lack of an experimental task in the neuropsychological examinations. Prior reports have shown that, on a task like the Iowa Gambling Task, the pattern of gambling in PPA is different from bv-FTD. ${ }^{25}$
The experimental task could have been useful to distinguish compulsion from gambling behavior.

To sum up, FTLD is a clinical-pathological syndrome that encompasses a heterogeneous group of neurodegenerative disorders. The different phenotypes described may 


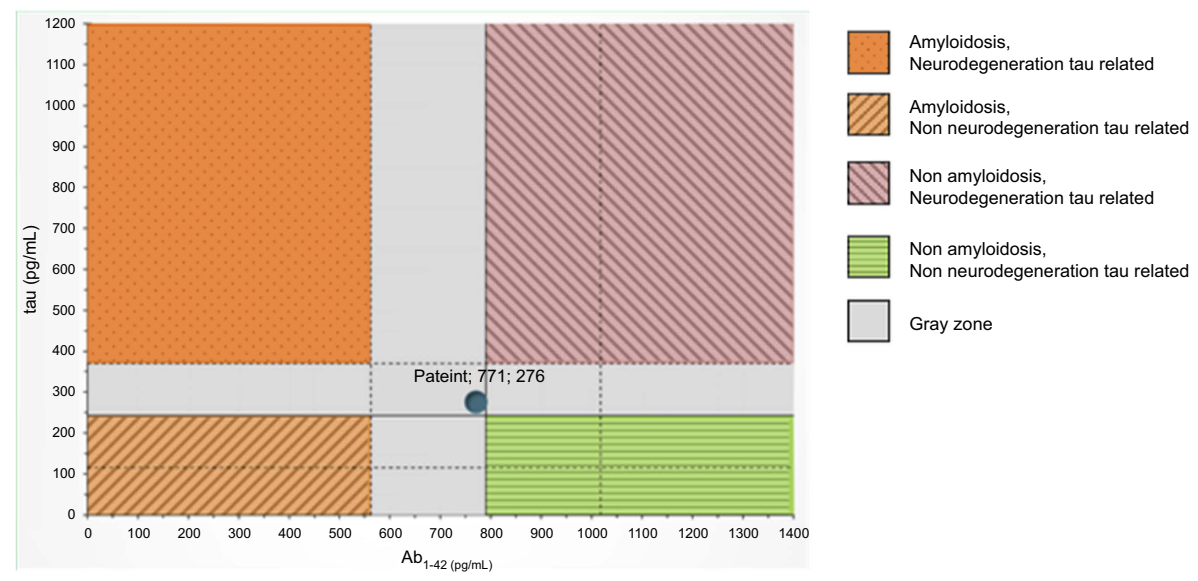

Figure 4 CSF examination within the normal range for age (A $\beta$ I-4277I pg/mL; tTau 276 pg/mL; nd 42.79 pg/mL; pTaul8I).

Abbreviations: MRI, magnetic resonance imaging; SPECT, single-photon emission computed tomography; CSF, cerebrospinal fluid.

have clinical and pathological characteristics that overlap, and it is possible that isolating a phenotype can be difficult because its borders may not be clearly evident as they may all belong to the same clinical-pathological spectrum.

\section{Conclusion}

In conclusion, this case is pertinent because of its atypical onset. Over the course of time, the evolution and progression of symptoms and the distributions of pathological changes suggest an underpinning FTLD pathology. It is worth noting that, in order to increase the accuracy of the clinical diagnosis, appropriate and validated batteries ${ }^{26}$ are essential since they are inexpensive and low-tech tools that can be used in a variety of clinical settings, such as primary, secondary or tertiary care and research studies.

\section{Consent}

Written informed consent was obtained from the patient for publication of this case report and accompanying images. This case study was approved by the institutional ethics committee of AO Pia Fondazione Card. G. Panico.

\section{Disclosure}

The author reports no conflicts of interest in this work.

\section{References}

1. Rohrer J, Geser F, Zhou J, et al. TDP-43 subtypes are associated with distinct atrophy patterns in frontotemporal dementia. Neurology. 2010;75:2204-2211. doi:10.1212/WNL.0b013e3181ed735b

2. Gorno-Tempini ML, Hillis AE, Weintraub S, et al. Classification of primary progressive aphasia and its variants. Neurology. 2011;76:1006-1014. doi:10.1212/WNL.0b013e31821103e6
3. Thompson SA, Patterson K, Hodges JR. Left/right asymmetry of atrophy in semantic dementia behavioral-cognitive implications. Neurology. 2003;61(9):1196-1203. doi:10.1212/01.WNL.0000091868.28557.B8

4. Gefen T, Wieneke C, Martersteck A, et al. Naming vs knowing faces in primary progressive aphasia: a tale of 2 hemispheres. Neurology. 2013;81(7):658-664. doi:10.1212/WNL.0b013e3182a08f83

5. Snowden JS, Thompson JC, Neary D. Knowledge of famous faces and names in semantic dementia. Brain. 2004;127(4):860-872. doi:10.1093/brain/awh099

6. Rosen HJ, Allison SC, Ogar JM, et al. Behavioral features in semantic dementia vs other forms of progressive aphasias. Neurology. 2006;67(10):1752-1756. doi:10.1212/01.wnl.0000247630.29222.34

7. Harris JM, Saxon JA, Jones M, Snowden JS, Thompson JC. Neuropsychological differentiation of progressive aphasic disorders. J Neuropsychol. 2018. doi:10.1111/jnp.12149

8. Manes FF, Torralva T, Roca M, Gleichgerrcht E, Bekinschtein TA, Hodges JR. Frontotemporal dementia presenting as pathological gambling. Nat Rev Neurol. 2010;6(6):347-352. doi:10.1038/nrneurol.2010.34

9. Tondo G, De Marchi F, Terazzi E, Sacchetti M, Cantello R. Frontotemporal dementia presenting as gambling disorder: when a psychiatric condition is the clue to a neurodegenerative disease. Cogn Behav Neurol. 2017;30(2):62-67. doi:10.1097/WNN.0000000000 000122

10. Catricalà E, Gobbi E, Battista P, et al. SAND: a screening for aphasia in NeuroDegeneration. Development and normative data. Neurol Sci. 2017;38:1469-1483. doi:10.1007/s10072-017-3001-y

11. Battista $P$, Catricalà E, Piccininni M, et al. Screening for aphasia in neuro degeneration for the diagnosis of patients with primary progressive aphasia: clinical validity and psychometric properties. Dement Geriatr Cogn Disord. 2018;46(3-4):243-252. doi:10.1159/000492632

12. Patton JH, Stanford MS, Barratt ES. Factor structure of the Barratt impulsiveness scale. J Clin Psychol. 1995;51(6):768-774. doi:10.1002/(ISSN)1097-4679

13. Lesieur HR, Blume SB. The South Oaks Gambling Screen (SOGS): A new instrument for the identification of pathological gamblers. $A m$ J Psychiatry. 1987;144:9.

14. Rascovsky K, Hodges JR, Knopman D, et al. Sensitivity of revised diagnostic criteria for the behavioural variant of frontotemporal dementia. Brain. 2011;134(9):2456-2477. doi:10.1093/brain/awr044

15. Brun A, Englund B, Gustafson L, et al. Clinical and neuropathological criteria for frontotemporal dementia. J Neurol Neurosurg Psychiatry. 1994;57(416):418.

16. Dubois B, Feldman HH, Jacova C, et al. Advancing research diagnostic criteria for Alzheimer's disease: the IWG-2 criteria. Lancet Neurol. 2014;13(6):614-629. doi:10.1016/S1474-4422(14) 70090-0 
17. McKeith IG. Consensus guidelines for the clinical and pathologic diagnosis of dementia with Lewy bodies (DLB): report of the Consortium on DLB International Workshop. J Alzheimers Dis Rep. 2006;9(s3):417-423.

18. Lanata SC, Miller BL. The behavioural variant frontotemporal dementia (bvFTD) syndrome in psychiatry. J Neurol Neurosurg Psychiatry. 2016;87(5):501-511. doi:10.1136/jnnp-2015-310697

19. Bang J, Spina S, Miller BL. Frontotemporal dementia. Lancet. 2015;386(10004):1672-1682. doi:10.1016/S0140-6736(15)00461-4

20. Seelaar H, Kamphorst W, Rosso SM, et al. Distinct genetic forms of frontotemporal dementia. Neurology. 2008;71::1220-1226. doi:10.1212/ 01.wnl.0000319702.37497.72

21. Park HK, Chung SJ. New perspectives on parkinsonism in frontotemporal lobar degeneration. J Clin Mov Disord. 2013;6(1):1. doi:10.14802/jmd.13001

22. Pan PL, Song W, Yang J, et al. Gray matter atrophy in behavioral variant frontotemporal dementia: a meta-analysis of voxel-based morphometry studies. Dement Geriatr Cogn Disord. 2012;33(23):141-148. doi:10.1159/000338176
23. Seeley WW. Selective functional, regional, and neuronal vulnerability in frontotemporal dementia. Curr Opin Neurol. 2008;21(6):701. doi:10.1097/WCO.0b013e3283168e2d

24. Clark L, Boileau I, Zack M. Neuroimaging of reward mechanisms in Gambling disorder: an integrative review. Mol Psychiatry. 2018;1. In Press.

25. Gleichgerrcht E, Torralva T, Roca M, et al. Decision making cognition in primary progressive aphasia. Behav Neurol. 2012;25(1):4552. doi:10.1155/2012/606285

26. Battista P, Miozzo A, Piccininni M, et al. Primary progressive aphasia: a review of neuropsychological tests for the assessment of speech and language disorders. Aphasiology. 2017;31:1359-1378. doi:10.1080/ 02687038.2017 .1378799
Clinical Interventions in Aging

\section{Publish your work in this journal}

Clinical Interventions in Aging is an international, peer-reviewed journal focusing on evidence-based reports on the value or lack thereof of treatments intended to prevent or delay the onset of maladaptive correlates of aging in human beings. This journal is indexed on PubMed Central, MedLine, CAS, Scopus and the Elsevier

\section{Dovepress}

Bibliographic databases. The manuscript management system is completely online and includes a very quick and fair peer-review system, which is all easy to use. Visit http://www.dovepress.com/ testimonials.php to read real quotes from published authors. 